\title{
Reversal of desferrioxamine induced auditory neurotoxicity during treatment with Ca-DTPA
}

\author{
B WONKE, ${ }^{*}$ A V HOFFBRAND, $\dagger$ M ALDOURI, $\dagger$ D WICKENS, $\ddagger$ D FLYNN, $₫$ M STEARNS, $\S$ \\ AND P WARNER§ \\ Departments of ${ }^{*}$ Haematology and $\ddagger$ Chemical Pathology, Whittington Hospital, and Departments of \\ †Haematology, \Paediatrics and \$Ear, Nose and Throat, Royal Free Hospital, London
}

SUMMARY Auditory neurotoxicity occurred in $13(26 \%)$ of 50 evaluable patients receiving long term desferrioxamine chelation. In five of these patients, all of whom were receiving high doses of desferrioxamine, the toxicity caused deafness. These five patients were treated with subcutaneous calcium diethylene triamine pentacetic acid (Ca-DTPA) with zinc supplements instead of desferrioxamine, and their hearing improved during periods of seven to 19 months. Their serum iron concentrations remained unchanged. We suggest that all patients receiving long term desferrioxamine should have audiometric assessments at 6-12 monthly intervals. Ca-DTPA with oral zinc supplements should be considered as alternative to desferrioxamine as an iron chelating treatment in patients with auditory neurotoxicity.

Iron overload is a well recognised complication of long term transfusion treatment. In 1963 desferrioxamine was first introduced and given both intramuscularly and intravenously. In 1976, however, the subcutaneous delivery of the drug by a portable infusion pump was introduced. ${ }^{12}$ During the past 10 years long term parenteral treatment of desferrioxamine at doses of $20-50 \mathrm{mg} / \mathrm{kg} /$ day has been used to maintain iron balance in patients on regular transfusion regimens with stores about five to 10 times normal.

Desferrioxamine is a comparatively non-toxic drug with few side effects. In recent years, however, high doses of desferrioxamine given either intravenously or subcutaneously were found to be toxic to the eyes and ears. ${ }^{4-7}$ In the present study the incidence of auditory abnormalities was assessed in a group of 54 patients with thalassaemia major who were receiving regular blood transfusions and long term treatment with subcutaneous desferrioxamine. In five patients, desferrioxamine treatment was withdrawn because of high tone deafness, and iron chelation was continued with calcium diethylene triamine pentacetic acid (Ca-DTPA). The effects of stopping desferrioxamine on the hearing of these patients have been assessed as well as the efficacy of Ca-DTPA compared with desferrioxamine as a long term iron chelator.

\section{Patients and methods}

Fifty four patients aged 5-35 (mean 19) years with homozygous $\beta$ thalassaemia were studied. There were 26 male and 28 female patients, and they had received from 53 to 608 (mean 326) units of blood. Forty nine patients were receiving $40-50 \mathrm{mg} / \mathrm{kg}$ body weight of desferrioxamine, and five patients 85-160 $\mathrm{mg} / \mathrm{kg}$ body weight subcutaneously over 8-12 hours on five or six nights each week.

In addition, the patients were given desferrioxamine 1-2 g/unit of blood intravenously at the time of transfusion. Oral vitamin C $100-200 \mathrm{mg}$ daily was added to this treatment. The duration of treatment with desferrioxamine ranged from two to nine years. Plasma zinc, copper, caeruloplasmin, magnesium, calcium, and 24 hour urinary iron and zinc concentrations were measured initially and then monthly in the five patients receiving treatment with Ca-DTPA.

All patients were tested audiometrically. Before testing, histories were taken to make sure the patients did not have middle ear disease or had had operations that might have affected inner ear function. All patients were asked about possible acoustic trauma, head injury, and the use of previously known ototoxic drugs (such as gentamicin); each patient was also examined and assessed. Four of the 54 patients were excluded because 
definite aetiological factors were identified-in particular, they reported that they had been exposed to continuous excessive amounts of sound or to ototoxic drugs. Pure tone audiometry was carried out in a sound proofed booth; masking was used when indicated and the equipment was calibrated to national standards. Bone conduction was tested when a hearing loss had been identified to indicate whether the loss was conductive or sensorineural.

Those patients with grossly abnormal responses to pure tone audiometry were tested by brain stem evoked response audiometry to identify the possible sites of the lesions. Patients were retested at six monthly intervals when possible. The Madsen OB 802 Clinical Audiometer was used for the pure tone audiometry and the Medelec ER 94A Sensor for the evoked response audiometry.

Serum ferritin concentrations were measured by an immunoradiometric assay, a modification of the method of Addison et al, ${ }^{8}$ and plasma zinc and sopper concentrations with a Perkin-Elmer model 306 atomic absorption spectrophotometer by a modification of the method of Scudder et al. ${ }^{9}$ Urinary zinc was estimated by direct aspiration of urine into an air/acetylene flame of a Perkin-Elmer model 306 atomic absorption spectrophotometer. Urine specimens giving absorptions greater than 15 $\mu \mathrm{mol} / 1$ were diluted $1: 20,1: 50$, or $1: 100$, as appropriate, and reaspirated. Caeruloplasmin concentrations were estimated by single radial immunodiffusion. ${ }^{10}$ Commercially prepared NOR partigen immunodiffusion plates, lyophilised protein standards, and controls were obtained from Hoeschst UK. Serum magnesium concentrations were analysed with a Perkin-Elmer model 370 atomic absorption spectrophotometer by a modification of the method of Hansen and Freier. ${ }^{11}$ Plasma calcium was analysed by a standard SMA-2 method (Technicon). CaDTPA was obtained from Astrapin Pharmaceutical Preparations and the MS-16 syringe driver from Graseby Dynamics.

\section{Results}

\section{AUDIOMETRY}

Thirteen of the evaluable patients $(26 \%)$ showed hearing loss (at least one point on the audiogram below 30 decibels). The hearing loss showed three different patterns. A high tone dip was found in six of the patients (12\%) (fig 1); all were asymptomatic. This is similar to the loss found in acoustic trauma, but none of these patients had been exposed to excessive noise. Two of the patients $(4 \%)$, also asymptomatic, had low tone hearing loss (fig 2). Five patients $(10 \%)$, all of whom were symptomatic had severe hearing loss (fig 2) that was most pronounced at high tones. One needed a hearing aid, another had difficulties at school, and the other three were aware of their problems and tried to compensate by sitting in the front rows at college. All five patients with severe hearing loss were receiving comparatively high doses of desferrioxamine (table 1), and four had normal or nearly normal serum ferritin concentrations (table 2). No appreciable abnormality other than a threshold shift was seen in these patients when they were tested by brain stem evoked audiometry, indicating that the lesions were probably in the cochlea rather than a more proximal position along the auditory pathway.

\section{TREATMENT}

Treatment with desferrioxamine was immediately stopped in the five symptomatic patients and replaced by Ca-DTPA. The dose of Ca-DTPA ranged
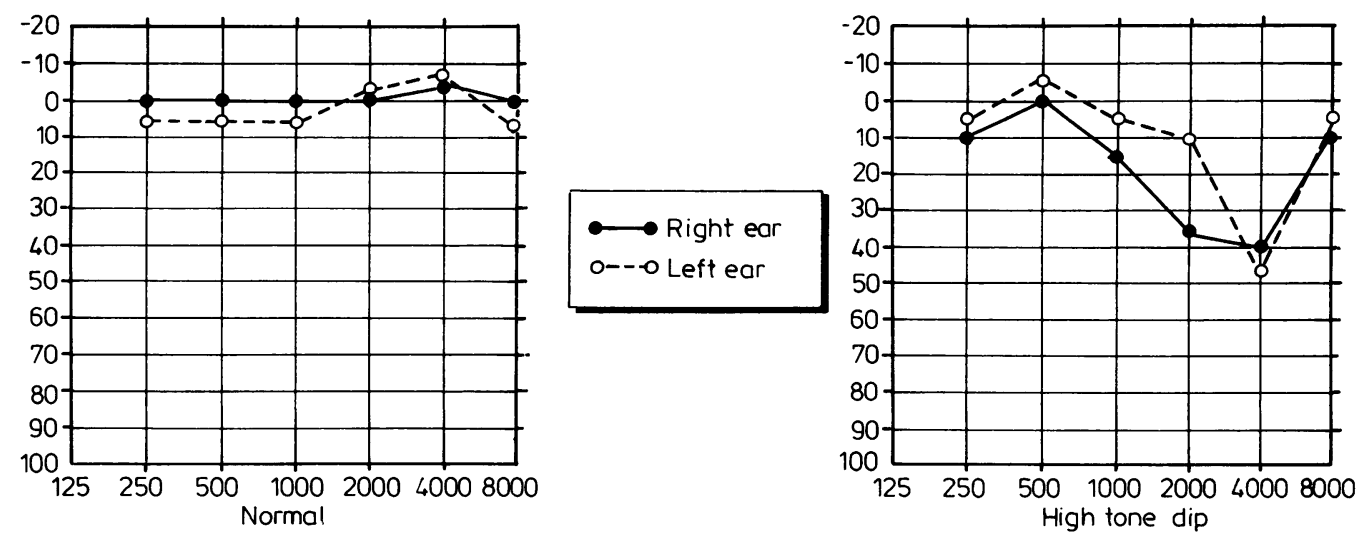

Fig 1 On the left, normal audiogram; on the right audiogram typical of the six patients $(12 \%)$ with high tone hearing loss. 

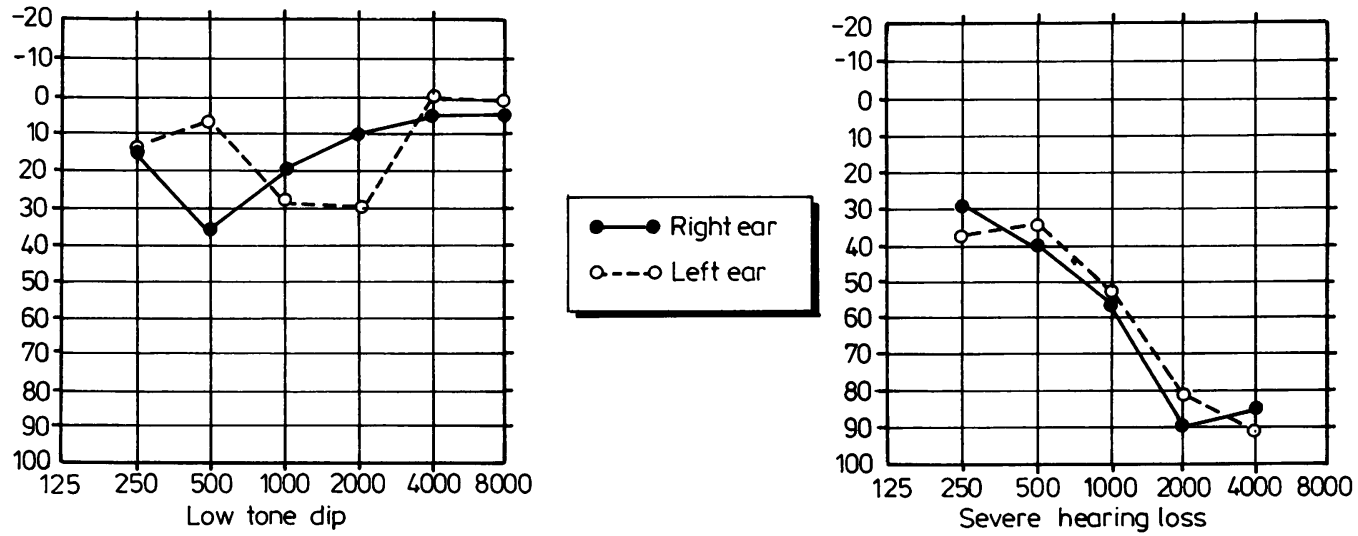

Fig 2 On the left, typical audiogram of two of the patients with low tone hearing loss. On the right typical audiogram of the five patients with severe deafness and loss of hearing at all frequencies, most pronounced at high tone.

initially from $0 \cdot 5 \mathrm{~g}$ (cases 4 and 5) to $1 \mathrm{~g}$ (cases 1,2 , and 3 ) given subcutaneously on alternate days. This was increased after two months in all cases to $1 \mathrm{~g}$ five nights each week and $1 \mathrm{~g}$ of Ca-DTPA/unit of blood transfused (table 2). Ca-DTPA was given subcutaneously by the infusion pump over a period of 10-12 hours. Zinc sulphate was given orally as 200 mg capsules or $220 \mathrm{mg}$ effervescent tablets two to five times daily depending on the plasma zinc concentration.

Table 2 gives details of the serum ferritin concen- trations in these patients before treatment with $\mathrm{Ca}$ DTPA was started, and the mean and range of serum ferritin measured every two to three months during treatment with Ca-DTPA. The normal ranges are (men) 39-340 $\mu \mathrm{g} / \mathrm{l}$ and (women) 14-148 $\mu \mathrm{g} / \mathrm{l}$. The serum ferritin concentrations tended to rise slightly on the initially low dose of Ca DTPA but fell to the concentrations recorded before treatment with Ca-DTPA when the frequency and dose of Ca-DTPA were increased. Urinary iron excretion in response to subcutaneous Ca-DTPA

Table 1 Details of five patients with severe deafness at the beginning of treatment with Ca-DTPA

\begin{tabular}{|c|c|c|c|c|c|}
\hline $\begin{array}{l}\text { Case } \\
\text { No }\end{array}$ & Sex & $\begin{array}{l}\text { Age } \\
\text { (years) }\end{array}$ & $\begin{array}{l}\text { No of units } \\
\text { transfused }\end{array}$ & $\begin{array}{l}\text { Duration of } \\
\text { desferrioxamine } \\
\text { treatment (years) }\end{array}$ & $\begin{array}{l}\text { Dose of } \\
\text { desferrioxamine } \\
\text { ( } \mathrm{mg} / \mathrm{kg} \text { body weight) }\end{array}$ \\
\hline 1 & Male & 20 & 302 & 8 & 60 \\
\hline 2 & Female & 18 & 313 & 9 & 80 \\
\hline 3 & Female & 17 & 297 & 7 & 85 \\
\hline 4 & Male & 8 & 108 & 5 & 90 \\
\hline
\end{tabular}

Table 2 Association between serum ferritin concentrations and treatment with Ca-DTPA

\begin{tabular}{|c|c|c|c|c|}
\hline \multirow[t]{3}{*}{ Case } & \multirow{3}{*}{$\begin{array}{l}\text { Duration of treatment } \\
\text { with Ca-DTPA } \\
\text { (months) }\end{array}$} & \multicolumn{3}{|c|}{ Serum ferritin concentration $(\mu g / l)$} \\
\hline & & \multirow{2}{*}{$\begin{array}{l}\text { Before treatment } \\
\text { with Ca-DTPA } \\
\text { Mean }\end{array}$} & \multicolumn{2}{|c|}{ During treatment with Ca-DTPA } \\
\hline & & & Mean & (Range) \\
\hline 1 & 7 & 1500 & 1540 & $(1300-2070)$ \\
\hline 2 & 8 & 380 & 400 & $(220-430)$ \\
\hline 3 & 9 & 646 & 760 & $(440-860)$ \\
\hline 4 & 12 & 180 & 340 & $(280-480)$ \\
\hline 5 & 19 & 400 & 800 & $(450-1100)$ \\
\hline
\end{tabular}


did not vary significantly over the period of treatment (table 3). All five patients had normal plasma zinc concentrations before starting on treatment with Ca-DTPA (table 4). Plasma zinc concentrations were low in patients 2,3 , and 5 in the first few months of treatment with Ca-DTPA. This was because the patients could not tolerate the zinc sulphate capsules, and was corrected by changing to the effervescent zinc sulphate tablets and temporarily increasing the dosage to five tablets daily. Urinary zinc excretion and plasma zinc concentrations increased substantially in these three patients when oral zinc supplementation was increased.

Table 3 Urinary iron excretion in response to treatment with Ca-DTPA

\begin{tabular}{|c|c|c|c|c|c|}
\hline \multirow{3}{*}{$\begin{array}{l}\text { Duration of treatment } \\
\text { with Ca-DTPA (months) }\end{array}$} & \multicolumn{5}{|c|}{ Urinary iron excretion ( $m g / 24$ hours) } \\
\hline & \multicolumn{5}{|c|}{ Case Nos } \\
\hline & 1 & 2 & 3 & 4 & 5 \\
\hline 0 (Control) & 0.51 & 0.43 & 0.76 & $0 \cdot 07$ & 0.07 \\
\hline 1 & $4 \cdot 0$ & $6 \cdot 3$ & $3 \cdot 7$ & $3 \cdot 4$ & $3 \cdot 4$ \\
\hline 2 & 6.4 & $7 \cdot 4$ & 4.9 & $4 \cdot 10$ & $2 \cdot 1$ \\
\hline 3 & $18 \cdot 6$ & $19 \cdot 8$ & $10 \cdot 4$ & $8 \cdot 4$ & 6.9 \\
\hline 4 & $25 \cdot 0$ & $26 \cdot 6$ & $10 \cdot 2$ & $8 \cdot 0$ & $*$ \\
\hline 5 & $20 \cdot 8$ & $24 \cdot 0$ & $*$ & $7 \cdot 4$ & $5 \cdot 6$ \\
\hline 6 & $17 \cdot 7$ & $34 \cdot 4$ & $7 \cdot 0$ & $7 \cdot 6$ & $6 \cdot 7$ \\
\hline 7 & $25 \cdot 1$ & $*$ & $6 \cdot 0$ & $8 \cdot 5$ & $*$ \\
\hline 8 & $*$ & $29 \cdot 3$ & $*$ & $6 \cdot 7$ & $5 \cdot 6$ \\
\hline 9 & $*$ & $*$ & $10 \cdot 0$ & $7 \cdot 6$ & $5 \cdot 6$ \\
\hline 10 & $*$ & $*$ & $*$ & $6 \cdot 6$ & $7 \cdot 3$ \\
\hline 11 & $*$ & $*$ & $*$ & $7 \cdot 1$ & $5 \cdot 7$ \\
\hline 12 & $*$ & $*$ & $*$ & $8 \cdot 6$ & $5 \cdot 3$ \\
\hline 19 & $*$ & $*$ & $*$ & $*$ & $6 \cdot 5$ \\
\hline
\end{tabular}

*Not measured.
Subsequently urinary and plasma zinc concentrations did not vary greatly among the five patients, with supplementation of two or three zinc sulphate capsules or tablets daily (table 4). Normal zinc concentrations are urine $4 \cdot 5-9 \cdot 0 \mu \mathrm{mol} / 24$ hours, and plasma $11.5-17 \mu \mathrm{mol} / \mathrm{l}$. There were no significant changes in plasma copper, caeruloplasmin, magnesium, or calcium concentrations during treatment with Ca-DTPA (unpublished observations).

All patients with severe hearing loss showed substantial improvement in hearing. One patient recovered completely after 19 months of treatment with Ca-DTPA (fig 3). Improvement was noticeable after three to four months on treatment in two other patients, the remaining three (including the patient mentioned above), making more gradual recoveries. In one patient the delay may have been caused by concomitant middle ear infection that required several courses of treatment with antibiotics.

\section{Discussion}

Desferrioxamine is a remarkably non-toxic drug with few side effects despite the large doses needed over many years. There have been local reactions to the drug and few patients develop allergic manifestations, but in most cases desensitisation can be successfully undertaken. ${ }^{12}$

In recent years, however, high doses of desferrioxamine given intravenously or subcutaneously have sometimes caused reversible retinal abnormalities, including cone and rod dysfunction, night blindness, field defects, and dyschromatopsia. ${ }^{5} 6$ Tinnitus and high frequency sensorineural hearing

Table 4 Concentrations of zinc in urine ( $\mu$ mol/24 hours) and plasma ( $\mu$ mol/l) during treatment with Ca-DTPA

\begin{tabular}{|c|c|c|c|c|c|c|c|c|c|c|}
\hline \multirow[t]{2}{*}{$\begin{array}{l}\text { Duration of treatment } \\
\text { with Ca-DTPA (months) }\end{array}$} & \multicolumn{2}{|c|}{$\begin{array}{l}\text { Case No } \\
l\end{array}$} & \multicolumn{2}{|l|}{2} & \multicolumn{2}{|l|}{3} & \multicolumn{2}{|l|}{4} & \multicolumn{2}{|l|}{5} \\
\hline & Urine & Plasma & Urine & Plasma & Urine & Plasma & Urine & Plasma & Urine & Plasma \\
\hline \multicolumn{11}{|c|}{0 (Before treatment with } \\
\hline Ca-DTPA) & $7 \cdot 2$ & $15 \cdot 0$ & $6 \cdot 1$ & $13 \cdot 4$ & $4 \cdot 6$ & $16 \cdot 7$ & $4 \cdot 3$ & $16 \cdot 8$ & $5 \cdot 3$ & $14 \cdot 1$ \\
\hline 1 & 227 & $20 \cdot 8$ & 160 & $13 \cdot 4$ & 142 & $12 \cdot 1$ & 214 & $19 \cdot 5$ & 51 & $11 \cdot 2$ \\
\hline 2 & 297 & $22 \cdot 2$ & 240 & $*$ & 160 & $5 \cdot 9$ & 113 & $16 \cdot 8$ & 50 & $14 \cdot 1$ \\
\hline 3 & 445 & $20 \cdot 0$ & $*$ & $*$ & 505 & $16 \cdot 7$ & 769 & $49 \cdot 2$ & 129 & $9 \cdot 4$ \\
\hline 4 & 419 & $22 \cdot 0$ & 445 & $9 \cdot 8$ & 366 & $12 \cdot 1$ & 306 & $*$ & 1002 & $27 \cdot 5$ \\
\hline 5 & 438 & $*$ & 520 & $7 \cdot 9$ & $*$ & $11 \cdot 1$ & 220 & * & 233 & 13.4 \\
\hline 6 & 769 & $22 \cdot 1$ & $*$ & $17 \cdot 9$ & 340 & 11.7 & $*$ & 48.4 & 167 & $*$ \\
\hline 7 & 425 & $*$ & 720 & 18.9 & $*$ & 19.4 & $*$ & $*$ & $*$ & $*$ \\
\hline 8 & $*$ & $*$ & 465 & $17 \cdot 9$ & 264 & $12 \cdot 7$ & 197 & $*$ & 270 & $*$ \\
\hline 9 & $*$ & $*$ & $*$ & $*$ & 320 & $12 \cdot 0$ & $*$ & $25 \cdot 4$ & 333 & $23 \cdot 5$ \\
\hline 10 & $*$ & $*$ & $*$ & $*$ & $*$ & $*$ & 200 & $*$ & 267 & $14 \cdot 6$ \\
\hline 12 & $*$ & $*$ & $*$ & $*$ & $*$ & $*$ & 230 & $23 \cdot 7$ & 287 & $13 \cdot 6$ \\
\hline 18 & $*$ & $*$ & $*$ & $*$ & $*$ & $*$ & $*$ & $*$ & 266 & $13 \cdot 6$ \\
\hline 19 & $*$ & $*$ & $*$ & $*$ & $*$ & $*$ & $*$ & * & $*$ & $12 \cdot 8$ \\
\hline
\end{tabular}

${ }^{*}$ Not measured. 

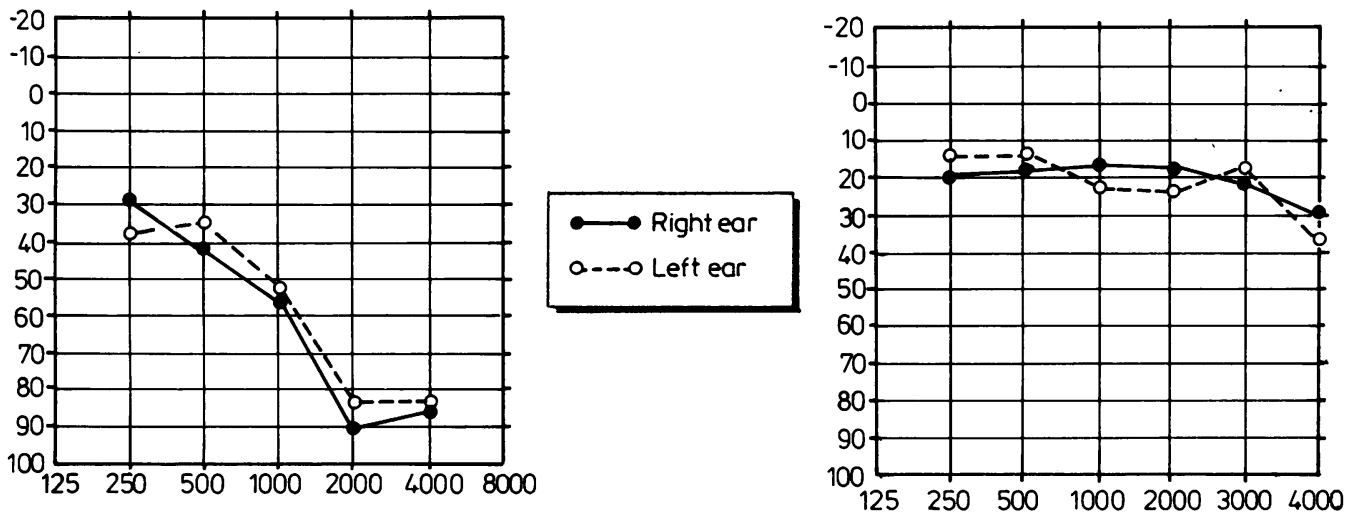

Fig 3 Audiograms of case 5 before and after 19 months treatment with Ca-DTPA.

deficits necessitating hearing aids have also occurred in some patients. ${ }^{4}$ Audiometric assessment in the present group of 50 patients with homozygous $\beta$ thalassaemia showed two patients with deafness and three patients with severe high frequency hearing loss; a further eight had mild asymptomatic hearing loss.

This incidence $(26 \%)$ of hearing loss is remarkably high, as none of these abnormalities would be expected in a group of healthy normal children. High frequency hearing loss is a characteristic finding in patients receiving desferrioxamine ${ }^{5}$ as well as other drugs-for example aminoglycosides. Although we cannot be certain it seems probable that the asymptomatic high tone 'dip' in six of our patients was caused by treatment with desferrioxamine. Mild 'low' tone hearing loss was also found in two patients, but the association of this with thalassaemia or treatment with desferrioxamine is not clear.

In all five severely affected patients treatment with desferrioxamine was stopped and replaced with Ca-DTPA. Ca-DTPA is as effective as desferrioxamine at inducing iron excretion. ${ }^{13}$ Our results show that iron excretion is maintained during long term treatment with Ca-DTPA and serum ferritin concentrations also remained steady despite continued blood transfusions, suggesting that Ca-DTPA was as effective as desferrioxamine at causing iron excretion in the long term.

Other side effects of Ca-DTPA include chills, fever, nausea, vomiting, diarrhoea, pruritis, and muscle cramps. ${ }^{14}{ }^{15}$ Pippard et al ${ }^{16}$ recently reviewed treatment with Ca-DTPA and found that if $\mathrm{Ca}$ DTPA was given subcutaneously it was as effective as desferrioxamine at chelating iron. They suggested that the zinc depletion induced by Ca-DTPA could be prevented by giving oral zinc supplements. Our present results confirm this, as none of our patients developed the well recognised signs of zinc deficiency-diarrhoea, apathy, cutaneous lesions, or hair loss-and plasma zinc concentrations were maintained within normal limits over prolonged periods of treatment.

In these studies Ca-DTPA given subcutaneously was well tolerated. Local irritation and thickening of the subdermal tissues of the injection sites did not occur. Oral zinc supplements caused nausea and vomiting at first in three of the five patients, but these symptoms disappeared as treatment continued and the three patients were changed to effervescent tablets. It was then possible to maintain plasma zinc concentrations within normal limits in all five patients despite continued treatment with $\mathrm{Ca}$ DTPA. Vitamin C supplements were not given as they do not seem to increase iron excretion with $\mathrm{Ca}$ DTPA. ${ }^{16}$

We conclude that the auditory toxicity caused by desferrioxamine is reversible in at least a proportion of patients, that Ca-DTPA in the doses that we used does not cause side effects on the cochlea, and that Ca-DTPA may be used effectively and safely for long term iron chelation. Oral zinc supplements and regular estimations of trace elements, however, are clearly needed during treatment.

We thank the United Kingdom Thalassaemia Society for generous financial support (MA).

\section{References}

1 Hussain MAM, Green N, Flynn DM, Hussein S, Hoffbrand AV. Subcutaneous infusion and intramuscular injection of desferrioxamine in patients with transfusional iron overload. Lancet 1976;ii:1278-80. 
${ }^{2}$ Propper RD, Cooper B, Rufo RF, et al. Continuous subcutaneous administration of desferrioxamine in patients with iron overload. $N$ Engl J Med 1977;197:418-23.

3 Aldouri MA, Wonke B, Hoffbrand AV, et al. Iron state and hepatic disease in patients with thalassaemia major, treated with long term subcutaneous desferrioxamine. J Clin Pathol 1987;40:1353-9.

${ }^{4}$ Marsh MN, Clark C, Holbrook JC, Shaffer JL. Tinnitus in a patient with beta thalassaemia on long term treatment with desferrioxamine. Postgrad Med J 1981;57:582-4.

5 Olivieri, NF, Buncic JR, Chew E, et al. Visual and auditory neurotoxicity in patients receiving subcutaneous desferrioxamine infusions. $N$ Engl J Med 1986;14:869-73.

6 Davies SC, Hungerford JL, Arden GB, Marcus RE, Miller MH, Huehns ER. Ocular toxicity of high dose intravenous desferrioxamine. Lancet 1983;ii:181-4.

${ }^{7}$ Rubinstein M, Dupont P, Doppee JP, Dehon C, Ducubu J, Hainaut J. Ocular toxicity of desferrioxamine. Lancet 1985; i: $817-8$.

8 Addison GM, Beamish MR, Hales CN, Hodgkins M, Jacobs A, Llewellin P. An immunoradiometric assay for ferritin in the serum of normal subjects and patients with iron deficiency and iron overload. J Clin Pathol 1972;25:326-9.

9 Scudder PR, Al-Timimi D, McMurray W, White AG, Zoob BC, Dormandy TL. Serum copper and related variables in rheumatoid arthritis. Ann Rheum Dis 1978;37:67-70.

${ }^{10}$ Marcini G, Carbonara AO, Heremans JF. Immunochemical quantitation of antigens by single radial immunodiffusion Immunochemistry 1985;2:235-42.

11 Hansen JL, Freier EF. The measurement of serum magnesium by atomic absorption spectrophotometry. American Journal of Medical Technology 1967;3:158-66.

12 Miller KB, Rosenwasser, LJ, Bessette JM, Beer DJ, Rocklin RE. Rapid desensitisation for desferrioxamine anaphylactic reaction. Lancet $1981 ; \mathrm{ii}: 1059$.

${ }^{13}$ Smith RS. Chelating agents in the diagnosis and treatment of iron overload in thalassaemia. Ann N Y Acad Sci 1964;119:77688.

${ }^{14}$ Graziano JH, Cerami A. Chelation therapy for the treatment of thalassaemia. Semin Hematol 1977;14:127-34.

15 Constantoulakis M, Economidou J, Karagiorga M, Katsanton A, Gyftaki E. Combined long term treatment of haemosiderosis with desferrioxamine and DTPA in homozygous beta thalassaemia. Ann NY Acad Sci 1974;232:193-200.

${ }^{16}$ Pippard MJ, Jackson MJ, Hoffman K, Petrou M, Modell CB. Iron chelation using subcutaneous infusions of diethylene triamine penta-acetic acid (DTPA). Scand J Haematol 1986;36:466-472.

Correspondence to Professor AV Hoffbrand, Department of Haematology, Royal Free Hospital, Pond Street, Hampstead, London NW3 $2 \mathrm{QG}$.

Accepted 26 July 1988 